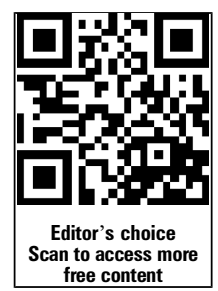

free content
free access mon
${ }^{1}$ Department of Neurology, Emory University School of Medicine, Atlanta, Georgia, USA

${ }^{2}$ CoAxia, Inc., Maple Grove, Minneapolis, Minnesota, USA ${ }^{3}$ NAMSA, Inc., Minneapolis, Minnesota, USA

${ }^{4}$ Wellstar Neurosurgery, Kennestone Hospital, Marietta, Georgia, USA

\section{Correspondence to} Dr Rishi Gupta, Wellstar Neurosurgery, 61 Whitcher Street, Suite 3110, Marietta, GA 30060, USA;

Rishi.gupta@wellstar.org

Received 21 January 2014 Revised 12 March 2014 Accepted 13 March 2014 Published Online First 7 April 2014

\section{CrossMark}

To cite: Belagaje SR

Zander K, Thackeray L, et al. J Neurolntervent Surg

2015:7:322-325.

\title{
Disposition to home or acute rehabilitation is associated with a favorable clinical outcome in the SENTIS trial
}

\author{
Samir R Belagaje, ${ }^{1}$ Kay Zander, ${ }^{2}$ Lisa Thackeray, ${ }^{3}$ Rishi Gupta ${ }^{4}$
}

\section{ABSTRACT \\ Objective To explore the impact of stroke severity and discharge disposition on 90-day outcomes in US patients enrolled in the SENTIS trial.}

Methods SENTIS is a previously published prospective randomized controlled trial. We analyzed the demographic information, National Institutes of Health Stroke Scores (NIHSS) on day 4, discharge disposition and 90-day modified Rankin Score (mRS). Univariate models were constructed to determine the impact of discharge disposition on 90-day outcomes as well as the association of the day 4 NIHSS with outcomes.

Results A total of 292 patients with a mean age of $65 \pm 14$ years were analyzed. Discharge disposition was distributed as follows: $153(52.1 \%)$ to an inpatient rehabilitation facility (IRF), 111 (38.0\%) to home and $28(9.6 \%)$ to a skilled nursing facility (SNF). Only 2 out of 28 patients $(7.1 \%)$ discharged to a SNF achieved a 90-day mRS $\leq 2$ compared with 60/153 (39.2\%) in the IRF group (OR 8.39 (95\% Cl 1.92 to 36.64), $\mathrm{p}=0.0047$ ). This association persisted after adjusting for age and admission NIHSS. Only 3 of 50 patients (6\%) with a NIHSS of $\geq 14$ at day 4 achieved a mRS $0-2$ at day 90.

Conclusions This analysis shows that discharge to an IRF is associated with better neurological outcomes than discharge to a SNF. Additionally, patients with a NIHSS of $\geq 14$ at day 4 are unlikely to achieve independent function.

\section{INTRODUCTION}

Much clinical research has focused on the acute treatment of ischemic stroke in the hope of improving clinical outcomes. One such example is SENTIS, a prospective multicenter single-blind randomized trial of the use of NeuroFlo technology compared with standard acute stroke therapy. ${ }^{1}$ The trial did not demonstrate a benefit of this technology compared with medical therapy as measured by its primary efficacy endpoint. Recent large clinical trials of endovascular reperfusion interventions have also failed to show a beneficial outcome of such treatments compared with standard medical therapies. $^{2}{ }^{3}$ Each of these studies measures outcomes at 90 days from stroke ictus, and much of this time is spent in post-hospital care. Thus, a potential confounder to the results of such studies may relate to discharge disposition and the degree of rehabilitation provided to patients.

Prior studies have demonstrated that patients' post-stroke outcomes can vary depending on the type and nature of post-stroke rehabilitation they receive. In particular, these studies focused on the outcomes following post-hospital discharge to an independent rehabilitation facility (IRF) compared with a skilled nursing facility (SNF) and, consistently, patients who went to an IRF had improved outcomes compared with a SNF. ${ }^{4-6}$ However, many of these studies were undertaken at a time when acute stroke treatment was not widely available or implemented. Furthermore, in the current US healthcare system, patients without insurance or family support may not be eligible for inpatient rehabilitation services despite meeting criteria from a physical or medical aspect.

Two questions therefore remain unanswered: the impact of discharge disposition following acute stroke interventions on the overall outcome and whether discharge disposition is an overall factor in emerging treatments. In this analysis we attempt to address these issues by analyzing the SENTIS trial and determining the role of discharge disposition. Specifically, we hypothesize that subjects with similar severity of strokes and medical comorbidites will have better 90-day outcomes if they are discharged to an IRF or home than to a SNF.

\section{METHODS}

We reviewed the database from a multicenter single-blind randomized trial (SENTIS), the results of which have been previously published. ${ }^{1}$ As part of the trial, data pertaining to patient demographics (age, gender, race, medical comorbidities), admission National Institutes of Health Stroke Scores (NIHSS), stroke severity, disposition and 90-day outcomes were prospectively obtained. Stroke severity was determined by NIHSS at day 4 and classified into three groups: NIHSS $<8,8-13$ and $\geq 14$. Disposition following acute hospital care was classified as home, IRF or SNF depending on the subject's disposition. For the purposes of this analysis, a favorable outcome was defined as a 90-day modified Rankin Score (mRS) of $\leq 2$.

The analysis was limited to subjects enrolled in US study sites. Subjects who participated outside the USA and those who died prior to discharge were excluded. Similarly, we excluded those subjects whose discharge disposition or 90-day outcome data were incomplete.

\section{Statistical analysis}

Data from SENTIS subjects for the cohort defined above were summarized. Continuous data were 
summarized using number of subjects, mean, median, SD and data ranges. Categorical data were summarized using counts and percentages. The association of disposition with a favorable outcome was examined using logistic regression models that were fit for the odds of the defined favorable outcome (ie, mRS $\leq 2)$. ORs, corresponding 95\% CIs and $\mathrm{p}$ values comparing disposition groups were obtained. These analyses were performed on the complete cohort and within each stroke severity group. Statistical significance was assessed at an $\alpha$ level of $<0.05$. Reported $\mathrm{p}$ values are two-sided. No adjustment for multiple comparisons was made. All statistical analyses were performed using SAS V.9.2 or later (SAS Institute, Cary, North Carolina, USA).

\section{RESULTS}

Of the total of 515 patients randomized in the trial, 292 were included in the current analysis as meeting the inclusion/exclusion criteria. The mean age was $65 \pm 14$ years, with the majority of subjects being male (53\%), white (79\%) or African-American (14\%). Table 1 shows full demographic data for the subjects. The discharge disposition for the 292 patients was distributed as follows: $153(52.1 \%)$ to an IRF, $111(38.0 \%)$ to home and $28(9.6 \%)$ to a SNF.

Table 2 shows the post-hospital discharge disposition stratified by stroke severity according to the day 4 NIHSS. Severity was stratified as NIHSS $<8$ in $166 / 290$ patients (57.2\%), NIHSS $8-13$ in $73 / 290$ patients $(25.2 \%)$ and NIHSS $\geq 14$ in $51 / 290$ patients (17.6\%); the day 4 NIHSS data were missing for two patients. Table 2 also shows that the stroke severity was related to the location of disposition. As expected, patients with a lower NIHSS were more likely to be discharged home while patients with a higher NIHSS were more likely to be discharged to a SNF. Most patients with a NIHSS of $\geq 8$ were discharged to an IRF.

As shown in table 3, only 2 of 28 patients (7.1\%) who were discharged to a SNF achieved a 90-day mRS of $\leq 2$ compared with 60/153 (39.2\%) discharged to an IRF (OR 8.39 (95\% CI 1.92 to 36.64$), p=0.0047)$. After adjusting for age and admission NIHSS, this association remained (OR 6.11 (95\% CI 1.29 to 8.81$), \mathrm{p}<0.02)$. Subjects who were discharged home also had significantly higher odds (OR 6.64 (95\% CI 3.74 to 11.81), $\mathrm{p}<0.0001$ ) of achieving a favorable 90 -day outcome compared with those who were discharged to an IRF.

In an attempt to better understand the relationship between stroke outcomes, discharge disposition, and stroke severity, further analysis was undertaken of each stroke severity subgroup. In the subgroup with NIHSS $<8$, a subject discharged home had better odds of good recovery than those who were discharged to an IRF (OR 3.20 (95\% CI 1.39 to 7.36), $\mathrm{p}=0.0063)$. There was a trend favoring IRF compared with $\mathrm{SNF}$ in this subgroup (OR 7.33 (95\% CI 0.71 to 75.27), $\mathrm{p}<0.093)$. Despite the lower NIHSS scores and less severe strokes, only one subject who was discharged to a SNF had a favorable recovery. For the group whose NIHSS was 8-13, only 1 of 10 patients (10\%) in the SNF group achieved a 90-day mRS of 0-2 compared with 13 of 56 (23.2\%) in the IRF group. This did not achieve statistical significance with regard to showing a difference between IRF and SNF in the NIHSS 8-13 subgroup. In the group of subjects with a NIHSS $\geq 14$, none of the subjects discharged to a SNF and 2 of 33 (6.6\%) discharged to an IRF were able to meet a functional recovery outcome (table 4).
Table 1 Subject characteristics $(\mathrm{N}=292)$

\begin{tabular}{|c|c|c|}
\hline \multicolumn{3}{|l|}{ Characteristics } \\
\hline \multirow[t]{3}{*}{ Age (years) } & Mean \pm SD & $65.6 \pm 14.3$ \\
\hline & Median & 67.7 \\
\hline & Range & $19.4-95.9$ \\
\hline \multicolumn{3}{|l|}{ Gender } \\
\hline Male & $\%(n / N)$ & $52.7 \%(154 / 292)$ \\
\hline Female & $\%(n / N)$ & $47.3 \%(138 / 292)$ \\
\hline \multicolumn{3}{|l|}{ Race/ethnicity } \\
\hline American Indian/Alaska Native & $\%(n / N)$ & $0.0 \%(0 / 292)$ \\
\hline Asian & $\%(n / N)$ & $1.0 \%(3 / 292)$ \\
\hline Black or African American & $\%(\mathrm{n} / \mathrm{N})$ & $14.4 \%(42 / 292)$ \\
\hline Hispanic or Latino & $\%(n / N)$ & $6.2 \%(18 / 292)$ \\
\hline Native Hawaiian/Pacific Islander & $\%(n / N)$ & $0.0 \%(0 / 292)$ \\
\hline White & $\%(\mathrm{n} / \mathrm{N})$ & $79.1 \%(231 / 292)$ \\
\hline \multirow[t]{3}{*}{ Time from symptom onset to baseline (h) } & Mean \pm SD & $7.7 \pm 2.8$ \\
\hline & Median & 7.3 \\
\hline & Range & $1.8-13.8$ \\
\hline \multirow[t]{4}{*}{ Baseline NIHSS } & $n$ & 292 \\
\hline & Mean \pm SD & $10.6 \pm 4.2$ \\
\hline & Median & 10.0 \\
\hline & Range & $2.0-19.0$ \\
\hline \multicolumn{3}{|l|}{ Side of infarct } \\
\hline Right & $\%(n / N)$ & $43.6 \%(123 / 282)$ \\
\hline Left & $\%(n / N)$ & $56.4 \%(159 / 282)$ \\
\hline Medical history & & $\mathrm{N}=292$ \\
\hline \multicolumn{3}{|l|}{ General history } \\
\hline Diabetes mellitus & $\%(\mathrm{n} / \mathrm{N})$ & $21.2 \%(62 / 292)$ \\
\hline Insulin-dependent diabetes & $\%(\mathrm{n} / \mathrm{N})$ & $7.5 \%(22 / 292)$ \\
\hline Non-insulin dependent diabetes & $\%(n / N)$ & $13.7 \%(40 / 292)$ \\
\hline $\begin{array}{l}\text { Diabetes mellitus or new diagnosis within } 30 \text { days } \\
\text { of enrollment }\end{array}$ & $\%(\mathrm{n} / \mathrm{N})$ & $32.5 \%(95 / 292)$ \\
\hline Hypertension & $\%(n / N)$ & $71.2 \%(208 / 292)$ \\
\hline $\begin{array}{l}\text { Hypertension or new diagnosis within } 30 \text { days of } \\
\text { enrollment }\end{array}$ & $\%(\mathrm{n} / \mathrm{N})$ & $79.1 \%(231 / 292)$ \\
\hline Hyperlipidemia & $\%(\mathrm{n} / \mathrm{N})$ & $52.1 \%(152 / 292)$ \\
\hline Current smoker & $\%(\mathrm{n} / \mathrm{N})$ & $28.8 \%(84 / 292)$ \\
\hline \multicolumn{3}{|l|}{ Neurological history } \\
\hline Cerebral ischemic infarct (stroke) & $\%(\mathrm{n} / \mathrm{N})$ & $15.8 \%(46 / 292)$ \\
\hline Transient ischemic attack & $\%(\mathrm{n} / \mathrm{N})$ & $11.3 \%(33 / 292)$ \\
\hline Seizure & $\%(\mathrm{n} / \mathrm{N})$ & $3.4 \%(10 / 292)$ \\
\hline \multicolumn{3}{|l|}{ Cardiovascular history } \\
\hline Atrial fibrillation & $\%(n / N)$ & $23.3 \%(68 / 292)$ \\
\hline $\begin{array}{l}\text { Atrial fibrillation or new diagnosis within } 30 \text { days } \\
\text { of enrollment }\end{array}$ & $\%(\mathrm{n} / \mathrm{N})$ & $28.4 \%(83 / 292)$ \\
\hline Valvular disease/replacement & $\%(n / N)$ & $6.2 \%(18 / 292)$ \\
\hline Peripheral vascular disease & $\%(n / N)$ & $5.5 \%(16 / 292)$ \\
\hline Percutaneous transluminal coronary angioplasty & $\%(n / N)$ & $9.2 \%(27 / 292)$ \\
\hline Coronary artery bypass graft & $\%(\mathrm{n} / \mathrm{N})$ & $10.6 \%(31 / 292)$ \\
\hline Myocardial infarction & $\%(n / N)$ & $12.0 \%(35 / 292)$ \\
\hline Angina & $\%(n / N)$ & $8.2 \%(24 / 292)$ \\
\hline
\end{tabular}

NIHSS, National Institutes of Health Stroke Scores.

\section{DISCUSSION}

In this analysis we demonstrate that post-hospital discharge disposition is associated with the 90-day neurological outcome following an acute stroke intervention. Compared with those who were discharged to a SNF, subjects who were discharged home or to an IRF were significantly more likely to have a favorable outcome. Moreover, only 3 of 50 patients (6\%) with NIHSS $\geq 14$ at day 4 achieved a favorable 90 -day outcome. This finding has an impact on anticipated outcomes in patients with larger 
Table 2 Distribution of discharge disposition in US patients stratified by National Institutes of Health Stroke Scores (NIHSS) at day 4

\begin{tabular}{lcccc}
\hline NIHSS & Home & IRF & SNF & Total patients \\
\hline$<8$ & $99(59.6 \%)$ & $63(38.0 \%)$ & $4(2.4 \%)$ & 166 \\
$8-13$ & $7(9.6 \%)$ & $56(76.7 \%)$ & $10(13.7 \%)$ & 73 \\
$\geq 14$ & $4(7.8 \%)$ & $34(66.7 \%)$ & $13(25.5 \%)$ & 51 \\
& 110 & 153 & 27 & 290 \\
\hline
\end{tabular}

IRF, independent rehabilitation facility; SNF, skilled nursing facility.

NIHSS and may point to employing a stratified outcome expectation in future trials.

An IRF is a facility designed to provide comprehensive multidisciplinary rehabilitation for up to $3 \mathrm{~h}$ per day under supervision of a physician trained in rehabilitation and a team of therapists in a variety of disciplines (physical therapy, occupational therapy, speech therapy). On the other hand, SNFs are facilities that provide less intensive therapy services. In a 2006 study of Medicare services, $78 \%$ of SNF patients received therapy ranging from 45 min per week to $12 \mathrm{~h}$ per week. ${ }^{4}$ Disposition is often determined by a patient's ability to participate in therapy and demonstrate consistent functional improvement towards returning home. Patients who are sent home already demonstrate an ability to remain safe at home and usually have fewer deficits and less severe strokes. Patients with more comorbidities and severe neurological illness are often discharged to a SNF, thus having a lower probability of favorable 3-month outcomes.

However, factors affecting discharge disposition decisionmaking are not limited to medical or physical ones. A recent publication of consecutive patients treated with endovascular therapy found that patients discharged to a SNF had worse outcomes than those discharged to an IRF. Of importance, the two groups were similar with regard to medical comorbidities as measured by APACHE, NIHSS, and age. ${ }^{7}$ These findings suggest that other factors such as family support and socioeconomic status may affect the decision tree.

In previous stroke epidemiological studies, socioeconomic status and the presence of family support have been implicated as factors in outcomes. ${ }^{8} 9$ While the exact reasons in those studies are not clear, it is possible that underinsured or uninsured patients lack access to IRFs and therefore are discharged to a SNF. Furthermore, current guidelines require that patients discharged to IRFs go home following their acute rehabilitation. This is not possible for patients who do not have family support to provide adequate supervision and continued rehabilitation support; therefore, in such instances, patients are sent to SNFs even though they are fully able to tolerate the intensity of an IRF.

Table 3 Distribution of overall outcomes in US patients based on discharge disposition

\begin{tabular}{lllll}
\hline & Home & IRF & SNF & Total patients \\
\hline mRS 0-2 & $90(59.2 \%)$ & $60(39.5 \%)$ & $2(1.3 \%)$ & 152 \\
mRS 3-6 & $21(15.0 \%)$ & $93(66.4 \%)$ & $26(18.6 \%)$ & 140 \\
& 111 & 153 & 28 & 292 \\
\hline
\end{tabular}

IRF, independent rehabilitation facility; mRS, modified Rankin Score; SNF, skilled nursing facility.
Table 4 National Institutes of Health Stroke Scores (NIHSS) and discharge disposition relative to outcomes

\begin{tabular}{lllll}
\hline NIHSS & Home & IRF & SNF & Total patients \\
\hline NIHSS $<8$ & & & & \\
mRS 0-2 & $86(88.7 \%)$ & $44(70.9 \%)$ & $1(25 \%)$ & 131 \\
mRS 3-6 & $11(11.3 \%)$ & $18(29.1 \%)$ & $3(75 \%)$ & 32 \\
& 97 & 62 & 4 & 163 \\
NIHSS 8-13 & & & & \\
mRS 0-2 & $2(28.6 \%)$ & $13(23.2 \%)$ & $1(10 \%)$ & 16 \\
mRS 3-6 & $5(71.4 \%)$ & $43(76.8 \%)$ & $9(90 \%)$ & 57 \\
& 7 & 56 & 10 & 73 \\
NIHSS $\geq 14$ & & & & \\
mRS 0-2 & $1(25 \%)$ & $2(6.1 \%)$ & $0(0.0 \%)$ & 3 \\
mRS 3-6 & $3(75 \%)$ & $31(93.9 \%)$ & $13(100 \%)$ & 47 \\
& 4 & 33 & 13 & 50 \\
\hline
\end{tabular}

IRF, independent rehabilitation facility; mRS, modified Rankin Score; SNF, skilled nursing facility.

Our findings are consistent with previous studies. In a study of 92 IRFs and SNFs from multiple centers in different states, Kramer et $a l^{6}$ showed that IRFs are more likely to produce improvements in Activities of Daily Living as well as discharge to the community. In a second study, stroke patients discharged to IRFs had a significantly higher functional recovery than those discharged to a SNF. ${ }^{5}$ Furthermore, in another study focusing on outcomes by motor function and cognitive abilities, IRFs were superior. ${ }^{4}$ The main issue with these studies is that they involved patients in an era where acute stroke care treatment was not widely available, standardized, and optimized. It is therefore likely that sicker patients with unfavorable prognoses were being discharged appropriately to a SNF and were not having a good prognosis.

In the second part of the analysis we attempted to see if the disposition benefit was associated with a particular stroke severity class. While the ORs seemed to indicate the benefit of home and IRF, the results were statistically insignificant and further conclusions cannot be inferred. Furthermore, the absence of a favorable outcome in the SNF population in the most severe group led to the inability to calculate ORs. The small sample size in the groups probably contributed to the lack of statistical significance.

The main implication of our study results pertains to the design of future acute stroke intervention trials. The SENTIS trial, from which our cohort data arise, failed to demonstrate its primary objective. ${ }^{1}$ Similarly, other recent acute stroke interventional trials have failed to show benefit. While the reasons for failure are unclear and are probably multifactorial, a common theme among the trials is that they do not account for the amount or nature of rehabilitation following the acute intervention. Our analysis has shown that the location of discharge disposition is associated with outcomes; we have also discussed the general reasons for how such discharge dispositions are reached. It is possible that patients who qualify for IRF placement from a disability standpoint may not get placed there due to socioeconomic factors and are rather placed in a SNF. They may receive less intense therapy, have less favorable outcomes, and consequently have an acute intervention shown artificially not to have benefit (type II error). Whether this has actually occurred in previous acute trials and the frequency are simply unknown as it has not been considered in previous analyses. Based on our results, we recommend that future acute stroke 
trials account for post-hospital discharge disposition by including discharge disposition as a patient characteristic to ensure there is no imbalance among the treatment arms. In addition, we recommend that acute trials maximize opportunities for IRF and home discharge for qualifying patients.

This exploratory analysis does have some limitations. The decision tree and factors for discharge disposition cannot be effectively ascertained in a retrospective manner and can be studied in the future. Furthermore, despite SENTIS being an international multicenter trial, this study focused on US centers only; there were too many heterogeneous factors and small sample sizes to effectively compare US discharge disposition outcomes with other countries. Third, the presence or absence of vascular occlusion was not available in the SENTIS study in addition to the final infarct volume. Both of these variables are associated with 90-day outcomes and could not be controlled for in this analysis. ${ }^{10}$ Finally, our study demonstrates an association, but it is possible that there are confounding variables which led to the results. Further research is required to identify and understand the role of any such confounders.

Our study of acutely treated patients provides an exploratory framework and supports data from prior studies. If one considers the entire spectrum of stroke care as a series of individual phases, there are opportunities to intervene in each phase and optimize stroke outcomes. Our study suggests that each phase must be optimized in order to maximize the overall outcome from stroke.

Contributors Conception of the idea: SRB, RG. Drafting of the manuscript: RG, SRB. Critical revision: KZ, LT. Statistical analysis: LT.

Funding The initial SENTIS trial was sponsored by CoAxia, a commercial entity that no longer exists.

Competing interests $\mathrm{KZ}$ was formerly employed by CoAxia. LT is a consultant for CoAxia. RG is a consultant for Stryker Neurovascular, Covidien, Rapid Medical and
CoAxia; Steering Committee for Stryker DAWN trial and Penumbra THERAPY trial; royalties from UpToDate; Associate Editor for Journal of Neurointerventional Surgery, Journal of Neuroimaging and Interventional Neurology.

Ethics approval Data from the SENTIS trial required IRB approval from participating centers.

Provenance and peer review Not commissioned; externally peer reviewed.

Data sharing statement The data are held by CoAxia, which was acquired by Zoll. Requests for unpublished data can be sent to Zoll directly.

\section{REFERENCES}

1 Shuaib A, Bornstein NM, Diener H-C, et al. Partial aortic occlusion for cerebral perfusion augmentation: safety and efficacy of NeuroFlo in Acute Ischemic Stroke trial. [Erratum appears in Stroke 2011;42:e632-3]. Stroke 2011;42: 1680-90.

2 Ciccone A, Valvassori L, Nichaletti M, et al. SYNTHESIS Expansion Investigators. Endovascular treatment for acute ischemic stroke. N Engl J Med 2013;368: 904-13.

3 Broderick JP, Palesch YY, Demchuk AM, et al. Endovascular therapy after intravenous t-PA versus t-PA alone for stroke. N Engl J Med 2013;368:893-903.

4 Deutsch A, Granger CV, Heinemann AW, et al. Poststroke rehabilitation: outcomes and reimbursement of inpatient rehabilitation facilities and subacute rehabilitation programs. Stroke 2006;37:1477-82.

5 Kane RL, Chen Q, Finch M, et al. Functional outcomes of posthospital care for stroke and hip fracture patients under Medicare. J Am Geriatr Soc 1998;46:1525-33.

6 Kramer AM, Steiner JF, Schlenker RE, et al. Outcomes and costs after hip fracture and stroke. A comparison of rehabilitation settings. JAMA 1997;277:396-404.

7 Belagaje SR, Chung-Huan JS, Nogueira RG, et al. Discharge disposition to skilled nursing facility after endovascular reperfusion therapy predicts a poor prognosis. J Neurointerv Surg 2015;7:99-103.

8 Putman K, De Wit L, Schoonacker M, et al. Effect of socioeconomic status on functional and motor recovery after stroke: a European multicentre study. J Neurol Neurosurg Psychiatry 2007;78:593-9.

9 Cox AM, McKevitt C, Rudd AG, et al. Socioeconomic status and stroke. Lancet Neurol 2006:5:181-8.

10 Sun CH, Nogueira RG, Glenn BA, et al. "Picture to puncture": a novel time metric to enhance outcomes in patients transferred for endovascular reperfusion in acute ischemic stroke. Circulation 2013;127:1139-48. 